\title{
Mindfulness and Affect-Network Density: Does Mindfulness Facilitate Disengagement from Affective Experiences in Daily Life?
}

\author{
Zarah Rowland $^{1}$ D $\cdot$ Mario Wenzel ${ }^{1}$
}

Published online: 21 February 2020

(C) The Author(s) 2020

\begin{abstract}
Objectives Mindfulness has been positively associated with affective well-being, which may be explained by improved affective disengagement from prior experiences.

Methods We used a dynamic network approach to represent temporal interconnections between specific affective states (i.e., network density) — with less interconnected states indicating better affective disengagement — to investigate whether mindfulness predicted a lower network density. One-hundred twenty-five undergraduate students $(M$ age $=22.87 ; S D=5.06$ years; $77.6 \%$ female) completed a 40-day ambulatory assessment, reporting on their affective experiences and state mindfulness six times a day, and either practiced mindfulness or were on a wait-list (control condition).

Results We found that neither the mindfulness training, $\beta=-.11, p=.222$, nor state mindfulness, $\beta=-.06, p=.484$, were associated with affective network density. However, given prior research indicating the importance of prior meditation experience, we found that participants with prior meditation experience reported lower affective network density when they reported higher state mindfulness than participants without meditation experiences, $\beta=-.20, p=.048$.

Conclusion The present study found that neither high levels of mindfulness nor a mindfulness training automatically improved affective disengagement in the form of lower network density. Rather, it seems that only those who were already familiar with practicing mindfulness and who were mindful in their daily life experienced easier disengagement from previous affective experiences. These findings may point toward a different, more complex, understanding of mindfulness in experienced meditators.
\end{abstract}

Keywords Dynamic network approach · Affect network density - Affective disengagement · Ambulatory assessment . Mindfulness training

Mindfulness-based programs have been successfully applied in a wide array of clinical and non-clinical settings (Carmody and Baer 2008; Kuyken et al. 2008; Semple et al. 2010), and these programs have been found to have beneficial effects on affective well-being, reducing anxiety, distress, negative affect, and the risk of depression relapse (for meta-analyses, see Khoury et al. 2013, 2015). While a large number of studies have mainly focused on examining whether mindfulness-

Electronic supplementary material The online version of this article (https://doi.org/10.1007/s12671-020-01335-4) contains supplementary material, which is available to authorized users.

Zarah Rowland

zarowlan@uni-mainz.de

1 Institute of Psychology, Johannes Gutenberg University Mainz, Mainz, Germany based programs are effective, there is a growing interest in understanding how mindfulness and mindfulness-based programs improve affective well-being. Several studies suggested that mindfulness may exert its beneficial effects on well-being by facilitating disengagement or recovery from previous negative affective experiences (e.g., Britton et al. 2012; Cho et al. 2017). The network approach offers a novel way to examine how mindfulness improves affective well-being by modeling temporal interconnections between several affective states (e.g., Bringmann et al. 2013). Given that the network approach views affective-wellbeing as a specific system of interacting affective experiences, it can be used to investigate the influence of mindfulness on the affect system (also called emotion system; Pe et al. 2015; Snippe et al. 2017). This approach then allows researchers to examine the disengagement from previous experiences in daily life.

Although diverging definitions exist, state and trait concepts of mindfulness commonly involve two main 
components (Bishop et al. 2004): (1) maintaining attention on the present moment to monitor and explore the nature of current experiences as they evolve and pass, and (2) a non-judgmental, accepting, and calm attitude toward these experiences, which can also be referred to as equanimity (Desbordes et al. 2015). While attention monitoring helps meditators to notice affective experiences and states as they arise, an accepting and calm stance makes it possible to stay even-minded and unbiased toward these experiences, regardless of whether they are pleasant, unpleasant, or neutral (Desbordes et al. 2015; Lindsay and Creswell 2017). In this way, affective experiences can unfold and pass in their own time with a mindful individual seeking neither to prolong nor suppress either pleasant or unpleasant experiences. Consequently, attention monitoring and an accepting attitude may help mindful individuals to notice and accept present experiences, thus facilitating the disengagement from affective states that might otherwise persist after a particular situation is already over (Desbordes et al. 2015).

To examine this disengagement from affective experience, research has focused on changing affect levels, reflecting the dynamic nature of affective experiences instead of assuming them to be static (Frijda 2007). In laboratory settings, participants are commonly confronted with affect-relevant stimuli to allow researchers to observe how their affective reaction and recovery unfolds over time (i.e., how the intensity of a specific emotion increases and decreases). For example, Broderick (2005) found that undergraduate students who received a brief induction to enhance state mindfulness, as compared to participants who received a distraction or a rumination induction, reported lower levels of dysphoric mood directly after seeing affect-relevant stimuli. In the same vein, Cho et al. (2017) found dispositional mindfulness to be positively associated with emotional recovery, in that participants who were more mindful than others reported less negative affect when watching a neutral picture that was presented immediately after a negatively valenced picture. Moreover, individuals in a mindfulness intervention group, in comparison to a control group, reported decreased negative affect after a laboratory-based stress-induction (in the form of a social stressor) from pre-to-post mindfulness intervention (Britton et al. 2012), indicating facilitated emotional recovery.

This promising evidence from laboratory studies has been complemented by studies examining associations between mindfulness and disengagement from prior affective experiences directly in a real-life setting, using a so called ambulatory assessment approach (Trull and Ebner-Priemer 2013; Wenzel and Kubiak 2018), to increase ecological validity (Shiffman et al. 2008). While laboratory studies define affective recovery as a reduced negative affect level from one time point to the next, affective inertia indicates how strongly related affective experiences are from one time point to the next (Kuppens et al. 2010; Suls et al. 1998). High levels in affective inertia can be interpreted as regulatory weakness (De HaanRietdijk et al. 2016), with a reduced ability to disengage from recent events resulting in previous experiences spilling over into the future (Suls et al. 1998). Evidence for this assumption has been provided by Koval et al. (2015), who found increased negative affect inertia to be associated with less negative affect recovery from negative stimuli in both a laboratory and a real-life setting.

Affective inertia seems to be driven by impaired disengagement from recent experiences, making inertia a prime candidate to examine associations between mindfulness and disengagement from previous affective experiences. High levels of negative affect inertia have been found to be associated with lower levels of dispositional mindfulness (Keng and Tong 2016; Rowland et al. 2018), more severe depressive symptoms (Koval et al. 2012), low self-esteem, and negative emotionality (Houben et al. 2015; Kuppens et al. 2010), pointing toward the relevant role that disengaging from previous affective experiences plays for individuals' affective well-being in daily life.

However, Pe et al. (2015) contended that inertia represents only the spill-over of a single affective state or an aggregated measure of positive or negative affect, and does not in fact capture interconnections between several successive affective experiences, which could fluctuate and interconnect in complex ways. This differentiation is important, as a single affective state may not only be influenced by the spill-over effect immediately preceding it, but also by several different affective states that were experienced and appraised at a prior time point (Pe and Kuppens 2012). As a following example shows, several specific affective states may be able to interact with each other over time. A person may first feel sad after a negative event has happened. Since this experience may be interpreted as being unpleasant, this person may develop anger, unintentionally directing negative feelings toward others. This in turn, may at first result in decreased sadness and may increase some sort of satisfaction at the next moment. At the same time, anger may also trigger anxiousness and guilt since the person did not want to hurt other people, which then again may increase the level of sadness at the following moment. However, the feeling of satisfaction may, at the same time, decrease the subsequent feeling of sadness.

A novel way to consider the complexity of affective experiences and to examine how mindfulness helps people to disengage from previous experiences is by using a dynamic network approach (e.g., Bringmann et al. 2013; Borsboom and Cramer 2013; Wichers 2014). In this approach, each single positive and negative affective experience is predicted by all other prior affective experiences, resulting in an interconnected affective system that underlies affective well-being (Bringmann et al. 2016b; Pe et al. 2015; Wigman et al. 2015). If an individual finds it difficult to disengage from previous affective experiences, the 
spill-over of previous affective experiences may impact the next moment's experiences, resulting in a strongly interconnected and rigid pattern of single affective (or mental) states. In such a case, the affective network would be defined by high density (Newman 2010), with strong temporal connections between single affective states (Bringmann et al. 2016b; Pe et al. 2015). In contrast, according to the concept of nonjudgmental acceptance or equanimity (Desbordes et al. 2015; Lindsay and Creswell 2017), mindful individuals should respond even-minded toward all pleasant, unpleasant, and neutral experiences in that they neither try to make their present experiences more or less intense. This means that mindful individuals should experience their sensations as they occur regardless of the quality of past experiences. Even if there was a strong negative association between previous sadness and following anger (which seems "better" than a positive association since anger decreased after sadness), it would still indicate that less anger depends on previously experienced sadness and therefore, the present feeling would have been largely determined by previous experiences instead of situational present experiences (Kuppens et al. 2010; Pe et al. 2015). However, mindful individuals should have had none or only very few temporal connections between their present feelings and previous feelings, indicating better affective disengagement and less spillover from prior experiences (e.g., Keng and Tong 2016). Thus, if an individual has a more open-minded present awareness and an accepting attitude toward affective experiences, this may be represented by less interconnected affective states within an affective network: In this scenario, all kinds of experiences-both pleasant and unpleasant - may be noticed and accepted as they unfold and pass. In this way, present affective experiences can become disengaged from prior affective experiences (i.e., have low density).

Recent evidence on affect network density further supported the important role that disengagement from prior affective experiences may play in affective well-being. Both depression and neuroticism are characterized by automatic negative thought patterns (e.g., Roelofs et al. 2008), which make it difficult to disengage from prior negative experiences. Thus, such thought and affect patterns would be reflected in stronger interconnected moment-to-moment affective experiences. This was in fact found to be the case in people who were diagnosed with a major depressive disorder, in comparison to people who did not carry a clinical diagnosis (Pe et al. 2015), as well as in people reporting high levels of neuroticism (Bringmann et al. 2016b).

A further study by Snippe et al. (2017) examined the impact of mindfulness-based cognitive therapy (MBCT; Segal et al. 2002) on dynamic affect networks consisting of five mental states-worried, down, cheerful, agitated, and insecure - in participants with depressive symptoms. The researchers assessed momentary mental states several times a day at one week before and one week after the intervention. They then compared the dynamic networks for the mindfulness and wait-list control condition at baseline and post-intervention, respectively. While they did not find any significant changes in network density in the mindfulness versus control condition (Snippe et al. 2017), it is possible that the null findings may have been due to the fact that their affective network model consisted of mostly negative mental states (i.e., worried, down, agitated, or insecure), with only a single positive state (cheerful). As previous research has shown the importance of the inter-connectivity between both positive and negative affective states when investigating network density in relation to health-relevant constructs, this approach may not have captured relevant differences (Bringmann et al. 2016b; Pe et al. 2015).

The present study aimed at examining underlying processes of a mindfulness training that may help to improve affective well-being in daily life. It was hypothesized that affect network density in participants who practiced mindfulness would be lower than in individuals who were in the control condition, reflecting the ability to more easily disengage from past affective experiences. Additionally, it was hypothesized that individuals who reported high levels of state mindfulness in daily life would report lower affect network density than those who reported low levels of state mindfulness.

\section{Method}

\section{Participants}

We enrolled a total of 137 undergraduate psychology students at the Institute of Psychology in Mainz with an age range of 18 to 49 years $\left(M_{\text {age }}=23.08\right.$ years, $\left.S D_{\text {age }}=5.04\right)$. A-priori power analysis was performed to find small effects, $d=.33$, with a power of $95 \%$ on our primary outcomes of state mindfulness and self-control (Rowland et al. 2016). Participants received course credit in exchange for study participation. If participants answered at least $80 \%$ of the daily prompts, they were entered in a prize draw for one of two 100 Euro online-shop vouchers, on top of their course credit. Inclusion criteria were sufficient understanding of the German language, an age between 18 and 65 years, ability to use a smartphone for studyrelated purposes, and informed consent. Exclusion criteria were any psychiatric diagnosis or mental and physical condition that might have conflicted with study participation.

Eleven participants $(8.0 \%)$ withdrew their study participation over the course of the study, and we had to exclude one participant $(0.7 \%)$ who showed a signal adherence below $33 \%$, resulting in a total of 125 participants $\left(M_{\text {age }}=\right.$ 22.87 years, $S D_{\text {age }}=5.06 ; 77.6 \%$ female). 


\section{Procedure}

This study was part of a larger parent study (Rowland et al. 2016) that investigated the effects of an ultra-brief mindfulness training on mindfulness and perceived self-control (Rowland et al. 2019). Additionally, the present study complemented a recent study's findings regarding associations between mindfulness and affect dynamics, such as affective instability, inertia, and affect valence switch (Rowland et al. 2018) by using the same data set to examine affective disengagement with a novel dynamic network approach that considers the interconnections between several single affective states (e.g., Bringmann et al. 2016b; Pe et al. 2015). The parent study was registered with clinicaltrials.gov (NCT02647801) and was approved by the local ethics research committee at the Institute of Psychology, Johannes Gutenberg University Mainz in Germany. The ethical standards of this study were in line with the declaration of Helsinki (World Medical Association 2013). A study protocol that described all measures in detail can be found online (Rowland et al. 2016). The results on the effectiveness of the training with regard to the primary and secondary outcomes (state mindfulness and perceived self-control, and dispositional mindfulness and self-control, respectively) were reported in Rowland et al. (2019).

Participants attended a first laboratory session (t0) and completed several self-report questionnaires regarding selfcontrol and mindfulness. Participants were then allocated to either mindfulness training (original $n=68$; final $n=61$ ) or a wait-list control condition (original $n=69$; final $n=64$ ) using a restricted randomization allocation procedure. During the following five weeks, all participants from both conditions attended weekly laboratory sessions (t1-t5) and filled out further self-report questionnaires. Only participants who were allocated to the mindfulness condition received the mindfulness training described in the section "Training." During the final laboratory sessions (t6), all participants repeated the baseline assessment.

The ambulatory assessment protocol started one day after the first laboratory session and took place for 40 consecutive days. Between 10 am and 8 pm, all participants received six randomly distributed signals via their smartphones, prompting them to report their momentary feelings and present situational circumstances. Signals were randomly distributed and presented using the application movisensXS, Version 0.8.4203 (movisens GmbH, Karlsruhe, Germany). All signal prompts were set to be at least $45 \mathrm{~min}$ and not more than $200 \mathrm{~min}$ apart. Since there were some technical issues that resulted in some irregular time intervals between signals, we had to exclude 1243 affect observations (4.14\%) and 679 missed observations (2.26\%) from our analyses (Rowland et al. 2018). On average, signals were $103.35(S D=34.34)$ minutes apart from each other.
Training Participants who were allocated to the mindfulness condition practiced mindfulness at five weekly sessions ( $\mathrm{t} 1$ t5). The mindfulness training was based on a training developed by Levinson et al. (2014), whereby participants practiced mindfulness using a computer-based guided breathing meditation for approximately $12 \mathrm{~min}$. At each session, a research assistant was present who gave information about the concept of mindfulness and introduced the individuals to the meditation exercise. Participants were instructed to continuously count their breath from 1 to 9 to practice focusing attention on the present moment. Participants were asked to press a specific key when they counted from breath one to eight, and then another key when they counted their ninth breath. When they miscounted their breaths and pressed an inaccurate key, they received a feedback sound in order to nonjudgmentally shift their attention back to the present moment (Levinson et al. 2014). After the meditation, the research assistant talked with the participants about their difficulties and experiences during the meditation practice. Additionally, participants were encouraged to practice mindfulness on their own at home through a breathing meditation and body scan (audio files provided via the movisens application) or an informal mindfulness task. Out of 68 participants who were originally allocated to the mindfulness training, 45 participants practiced on average 4.2 times at home by listening to the meditation or body scan audio file (Rowland et al. 2019).

\section{Measures}

State measures We assessed affect in terms of eight common emotional states (e.g., Kuppens et al. 2010), which were based on the affective circumplex model (Russell 2003). Negative affect was assessed by asking participants to report their current level of feeling angry, anxious, depressed, and sad; and positive affect was assessed by asking participants to report their current level of feeling happy, excited, relaxed, and satisfied. Six times a day for 40 days, participants were asked to report their current affective state for each of these eight emotions on a visual slider scale from 0 (not at all) to 100 (very much). Each affect variable was within-person centered by subtracting the respective individual mean from the present moment affect value before estimating fixed effects for the population network.

Since we were not aware of any validated alternate state mindfulness scales when we were planning the study, we used the state version of the Mindful Attention Awareness Scale (MAAS; Brown and Ryan 2003) to measure state mindfulness. The original state version of the MAAS consists of five items. In this present study, we shortened the original version to three items to reduce participants' burden. We chose those three items that showed the highest eigenvalues during a factor analysis in our previous investigations. Participants were asked to indicate on a scale from 0 (not at all) to 6 (very much) 
to what degree they were paying full attention to what they had done since the last prompt. We first calculated an average state mindfulness score for each assessment time point and then aggregated these scores across all assessment time points for each individual to yield individual means of state mindfulness reports (Friese and Hofmann 2016).

Control measures At the beginning of the study ( $\mathrm{t} 0$ ), participants were asked to report whether they practiced yoga $(0=$ no; $1=$ yes $)$ and whether they practiced some sort of meditation $(0=n o ; 1=y e s)$. We created a new dummy coded variable called "previous meditation experience," coding 0 for all participants who practiced neither meditation nor yoga $(n=$ $102,81,6 \%)$, and 1 for participants who practiced yoga or some kind of meditation $(n=23,18.4 \%)$.

\section{Data Analyses}

We used the statistical software Stata 15 (StataCorp 2017) to calculate descriptive statistics and hierarchical multiple regressions. Additionally, we used the software $\mathrm{R}$ version 3.6.1 (R Core Team 2019) to estimate individual affect networks (graphicalVAR; Epskamp 2018) and to visualize population networks for each condition (qgraph; Epskamp et al. 2012). Data and syntax are available under https://osf.io/ jmz2n/.

Data preparation First, to make sure that our data were stationary (which is necessary when using time-series analyses to construct temporal networks), we tested all affect items for unit roots in our data set using the Fisher-type test over the whole 40-day assessment period (Augmented Dickey-Fuller [ADF] unit-root test; Choi 2001). This ADF unit-root test tests against the null hypothesis that all individual panels contain a unit root, a stochastic trend indicating an unpredictable systematic pattern. Since we could assume stationarity (all $Z^{-1}<43.38$, all $p$ 's $<.001$ ), we decided to construct temporal affect networks for each individual over the whole study period (240 possible observations per individual). We refrained from estimating weekly networks, which would have most likely yielded unreliable estimates due to the relatively small number of observations per week (a maximum of 42). This number of estimates falls below the usually used minimum of 50 to 70 observations per individual needed to construct dynamic networks (Bringmann et al. 2016b; Pe et al. 2015; Wigman et al. 2015).

We did not estimate associations between the last assessment of a day and the first assessment of the following day since the time interval differed greatly from within-day signal time intervals: temporally equally spaced measurements are an important assumption in order to model autoregressive models (Bringmann et al. 2013). Regardless of that, it is also assumed that emotion regulation occurs, and the brain is "reset" while sleeping (Gruber and Cassoff 2014) so that examining associations between evening and morning assessments would qualitatively differ from within-day associations. Thus, we fitted saturated graphical vector autoregression (GVAR; Wild et al. 2010) models that estimated all possible consecutive temporal within-day associations between all affective states for each individual to construct individual temporal networks over the whole study period. It is possible to use the least absolute shrinkage and selection operator regularization (LASSO; Tibshirani 1996) in combination with the extended Bayesian information criterion (EBIC; Chen and Chen 2008) on each individual network to obtain a sparse network structure (Epskamp et al. 2018). However, since we were interested in calculating network density as the average of all possible connections (Bringmann et al. 2016b; Pe et al. 2015) and not only the network connections of a sparse network, we only used unregularized models. Using GVAR, we computed temporal and contemporaneous networks for each individual, where estimates can be seen as edges that connect all eight affective network nodes (Bringmann et al. 2013). Here, we focus on the temporal network, where all affective states at time point $t$ were regressed on all affective states that were assessed at the prior measurement occasion $t-1$ for each respective individual (betas have been standardized to partial directed correlations; PDC; Wild et al. 2010). The results of the contemporaneous networks, which consisted of all associations at the same measurement time point (kappas have been standardized to partial contemporaneous correlations; PCC; Wild et al. 2010), can be found in the Supplementary Materials (Table S1-Table S4).

We then calculated the density of the individual temporal network of each individual (Bringmann et al. 2016b; Pe et al. 2015; Snippe et al. 2017; Wigman et al. 2015). Density refers to how interconnected all affect states are and therefore mirrors how self-predictive and rigid the whole affect system is (Pe et al. 2015). To calculate individual overall affect (OA) network density, we averaged over all absolute PDC values for each individual, indicating how strongly all affective states were associated with each other irrespective of negative or positive associations. We also calculated positive affect (PA) and negative affect (NA) density. We calculated PA density by averaging over the 32 absolute PDC values that represented estimates of all affective experiences at time point $t-1$ that predicted positive affective experiences at time point $t$; e.g., sad (NA) and relaxed (PA) at the previous time point predicted happy (PA) at the next time point. PA density indicates how much prior affective experiences (either negative or positive) predict momentary positive affective states (Pe et al. 2015). In a similar way, we calculated NA density by averaging over the 32 absolute PDC values that represented estimates of all affective experiences at time $t-1$ that predicted only negative experiences at time $t$. Thus, NA density indicates how much 
prior affective states predict momentary negative affective states (Pe et al. 2015).

Since contemporaneous networks do not include directed associations, the undirected associations between PA and NA states would be entered into the calculation of both the PA and NA network density. To account for that, we calculated contemporaneous network density differently: PA network density was calculated by averaging over all PCC absolute values that represented associations between PA states only, and NA network density was calculated by averaging over all PCC absolute values that represented associations between NA states only. Additionally, we calculated PA-NA network density, which was calculated by averaging over all PCC absolute values that represented associations between PA and NA states only. Associations between all temporal and contemporaneous network density parameters can be seen in the Supplementary Materials (Table S1).

Preliminary analyses Adherence with the ambulatory assessment protocol was calculated by dividing the number of completely answered prompts by all possible prompts for each individual. The individual adherence scores were then averaged over all participants. After that, we calculated descriptive statistics of all network density indices and mindfulness measures, and correlated individuals' means with each other.

Main analyses As an overview, we first graphically illustrated the population network of all individuals, which is based on the pooled data of all individual networks. The population network shows all possible connections, representing population-level OA network density.

We then used a hierarchical multiple regression to examine associations between temporal OA network density and both mindfulness measures (mindfulness training and the individual means of state mindfulness reports). In a first step, we regressed the individual OA network density values on the grand mean centered individual means of state mindfulness reports and condition allocation $(0=$ control condition; $1=$ mindfulness training). In a second step, we controlled for previous meditation experience $(0=$ no meditation or yoga experience; 1 = meditation or yoga experience).

To further examine whether mindfulness measures were associated with both PA and NA network density, we conducted two more hierarchical multiple regression analyses in which both mindfulness measures predicted PA and NA network density. Again, we added the control variable of previous meditation experience in a second step.

Additional analyses Since Baer et al. (2008) found that some mindfulness facets may only show associations with wellbeing in people with meditation experience, and not in individuals who only indicate that they are more mindful than others, we also tested whether previous meditation experience might moderate the relationship between the mindfulness measures and network density indices. In a third step, we added previous meditation experience as a moderator to our hierarchical multiple regression models by regressing the network density indices on each mindfulness measure, previous meditation experience, and the respective two-way interaction between the mindfulness measure and previous meditation experience.

\section{Results}

\section{Adherence}

Our final sample $(N=125)$ answered $75.9 \%$ of the AA protocol affect assessment prompts $(S D=14.8$, ranging between $34.6 \%$ and $97.5 \%$ ). Since we had to exclude those affect observations that were not prompted within the set time frame (see 'Procedure'), and we only included consecutive observations to our analyses, we had a final number of 14,257 observations. Descriptive statistics and inter-correlations of the temporal network density and all mindfulness measures are presented in Table 1.

\section{Associations between Network Density and Mindfulness}

Figure 1 shows the population network, which is based on the pooled data of all individuals. We also illustrated PA network density (all affective states predicting PA states) and NA network density (all affective states predicting NA states) by masking NA or PA network density based on the example of Pe et al. (2015), respectively. This allowed us to better understand how PA and NA states were interconnected with each other in general. Tables 2 and 3 show the main results of the hierarchical multiple regressions that examined associations between the mindfulness training and individual means of state mindfulness reports, as well as between OA, PA, and

Table 1 Descriptive statistics and inter-correlations between temporal network indices (OAD, NAD, PAD) and individual means of state mindfulness reports

\begin{tabular}{lllllll}
\hline & $M$ & $S D$ & Range & 1 & 2 & 3 \\
\hline 1 OAD & 0.10 & 0.02 & $0.06-0.15$ & & & \\
2 NAD & 0.10 & 0.02 & $0.05-0.17$ & $.85^{* *}$ & & \\
3 PAD & 0.10 & 0.02 & $0.05-0.16$ & $.88^{* *}$ & $.49^{* *}$ & \\
4 State Mindfulness & 4.76 & 0.88 & $2.79-5.99$ & -.05 & -.01 & -.07
\end{tabular}

Note. $\mathrm{OAD}=$ Overall affect network density; $\mathrm{NAD}=$ Negative affect network density; $\mathrm{PAD}=$ Positive affect network density; State mindfulness = individual means of State Mindful Attention Awareness Scale reports

$* p<.05$

$* * p<.001$ 


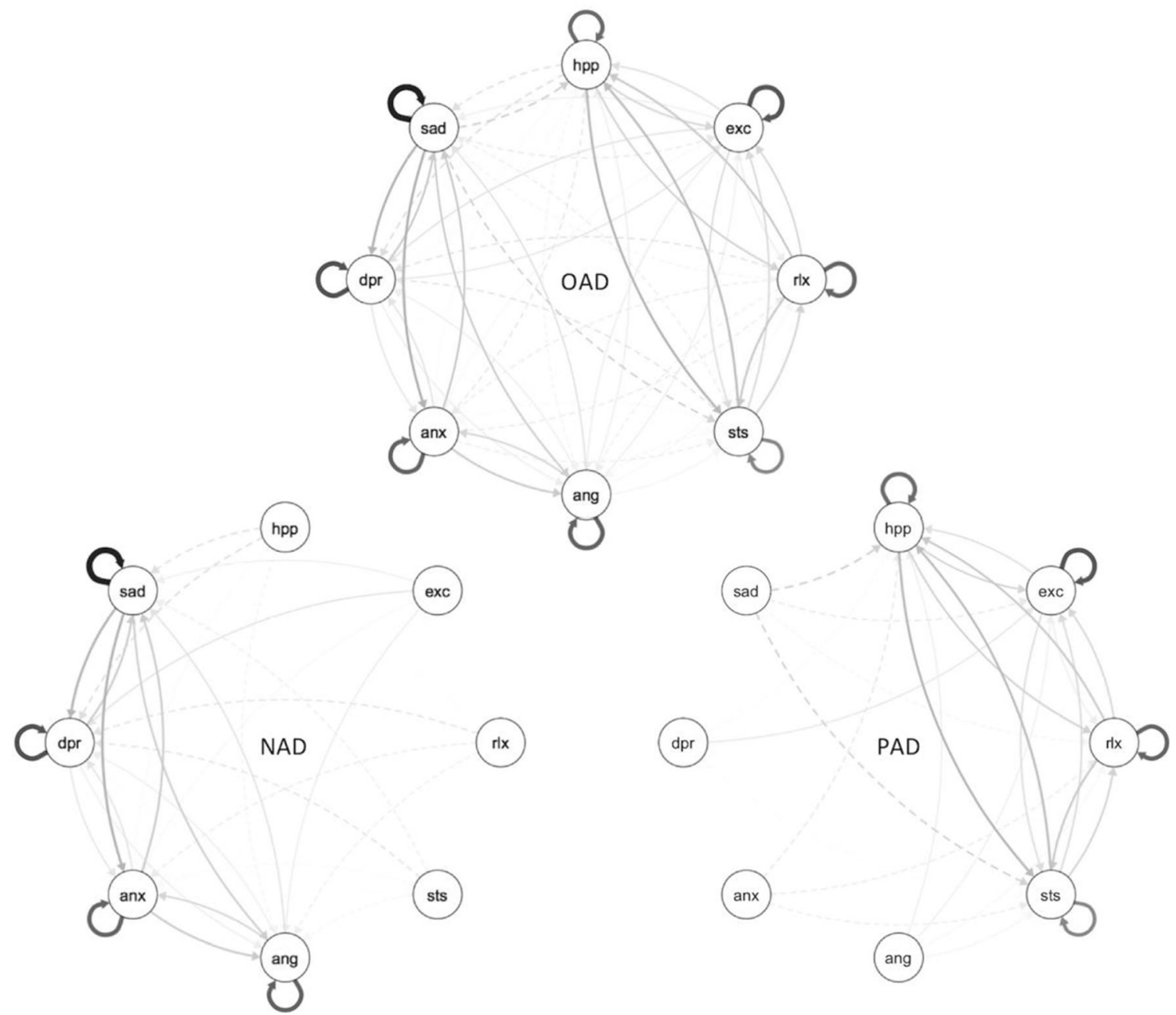

Fig. 1 Population network showing overall affect network density (OAD; upper), negative affect network density (NAD; lower, left side), and positive affect network density (PAD; lower, right side) of all 125 individuals. Arrows represent directed associations between a prior affective experience $(t-1)$ and an affective experience at the next moment $(t)$. Stronger associations are represented by bold and dark

NA temporal affect network density. Under Step 1, associations between each mindfulness measure and OA (Table 2), and between PA and NA network density (Table 3) are presented, without adjusting for any other control variables.

As can be seen in Table 2, Step 1, neither participants in the mindfulness training nor participants who reported high levels of state mindfulness showed significant associations with their OA temporal network density. Furthermore, neither PA nor NA network density were significantly predicted by either the individual means of all state mindfulness reports or mindfulness training (Step 1, Table 3). In a next step (Step 2), although we controlled for previous meditation experience, no changes were observed in the results presented under Step 1 (Tables 2 and 3). This was also found for all contemporaneous network indices (Tables S2, S3, and S4).

Next, since individuals with prior meditation experience tend to report different associations between mindfulness lines while weak associations are represented by thin and lightly colored lines. Positive associations are indicated by solid lines and negative associations are represented by dashed lines. Hpp = happy; exc = excited; $r l x=$ relaxed; sts $=$ satisfied; ang $=$ angry; anx $=$ anxious; $\mathrm{dpr}=$ depressed; $\mathrm{sad}=\mathrm{sad}$

and well-being (Baer et al. 2008), we tested whether prior experiences with meditation may moderate the associations between mindfulness measures and temporal affect density indices (Step 3): When we added both interaction terms between the mindfulness measures and prior experience to the model that predicted OA network density, we found that the model explained more variance (increasing from $1.7 \%$ to $5.3 \%$, but not significantly $p=.110$ ) in the outcome OA network density (Step 3, Table 2). Furthermore, the interaction term between the individual means of state mindfulness reports and prior meditation experience reached significance with a small negative effect $(\beta=-.200)$, indicating that participants who reported prior experiences with yoga or mediation and who reported high levels of state mindfulness also reported a lower OA network density in comparison to those who had no such prior experience and who indicated having high levels of mindfulness (Fig. 2). However, prior meditation 
Table 2 Overall affect network density (OAD) predicted by training, individual means of state mindfulness reports, and previous meditation experience (PME)

\begin{tabular}{|c|c|c|c|c|c|c|c|c|c|c|c|c|}
\hline \multirow[b]{2}{*}{ Predictors } & \multicolumn{4}{|l|}{ Step 1} & \multicolumn{4}{|l|}{ Step 2} & \multicolumn{4}{|l|}{ Step 3} \\
\hline & $B(S E)$ & $p$ & $\beta$ & $R^{2}$ & $B(S E)$ & $p$ & $\beta$ & $R^{2}$ & $B(S E)$ & $p$ & $\beta$ & $R^{2}$ \\
\hline Training & $-.004(.003)$ & .222 & -.112 & .014 & $-.004(.003)$ & .262 & -.104 & .017 & $-.002(.004)$ & .587 & -.055 & .053 \\
\hline State Mindfulness & $-.001(.002)$ & .484 & -.064 & & $-.001(.002)$ & .477 & -.065 & & $.001(.002)$ & .771 & .029 & \\
\hline PME & & & & & $-.002(.004)$ & .583 & -.050 & & $.000(.007)$ & .998 & .000 & \\
\hline Training x PME & & & & & & & & & $-.005(.009)$ & .554 & -.093 & \\
\hline State Mindfulness x PME & & & & & & & & & $-.010(.005)$ & .048 & -.200 & \\
\hline
\end{tabular}

Note. Step 1: Associations between OAD and mindfulness measures (Training and State mindfulness). Step 2: Controlled for PME. Step 3: Two-way interaction terms between PME and each mindfulness measure (Training and State mindfulness). OAD= Overall affect network density; State Mindfulness $=$ Grand-mean centered individual means of state Mindful Attention Awareness Scale reports; PME $=$ Previous meditation experience $(0=$ no meditation or yoga experience; $1=$ previous meditation or yoga experience $)$; Training $=$ Condition allocation $(0=$ control condition; $1=$ mind fulness training)

experience did not significantly moderate the effect of mindfulness training on OA network density.

With regard to NA and PA network density, the interaction term between the individual means of state mindfulness reports and prior experience in meditation (PA: $\beta=-.157$; NA: $\beta=$ $-.189)$ and between mindfulness training and prior mediation experience (PA: $\beta=-.206$ ) also showed small but nonsignificant negative effects. These negative effects were similar to those found regarding OA network density and the individual means of state mindfulness reports, indicating that participants with prior meditation experience relative to unexperienced participants also tended to report lower PA network density when they were high in state mindfulness and were allocated in the mindfulness training, and lower NA network density when they were more mindful. With regard to all conteporaneous network indices, all interaction terms did not reach significance (Step 3, Tables S2, S3, and S4).

\section{Robustness of Results}

Since yoga practice comes in many varieties and does not always involve actual meditation, we further tested whether our results were robust when only referring to those individuals as experienced meditators who reported to solely practice meditation $(n=9)$ or meditation and yoga $(n=10)$, but not those who only practiced yoga $(n=4)$. In that case, the found interaction effect of state mindfulness and previous meditation experience on OAD $(\beta=-.209, p=.038)$ and $\operatorname{NAD}(\beta=$ $-.222, p=.029)$ increased (Tables S5 and S6).

Table 3 Positive and negative affect network density (PAD and NAD) predicted by training, individual means of state mindfulness reports, and previous meditation experience (PME)

\begin{tabular}{|c|c|c|c|c|c|c|c|c|c|c|c|c|c|}
\hline \multicolumn{2}{|c|}{ Affect network density } & \multicolumn{4}{|l|}{ Step 1} & \multicolumn{4}{|l|}{ Step 2} & \multicolumn{4}{|l|}{ Step 3} \\
\hline Outcome & Predictors & $B(S E)$ & $p$ & $\beta$ & $R^{2}$ & $B(S E)$ & $p$ & $\beta$ & $R^{2}$ & $B(S E)$ & $p$ & $\beta$ & $R^{2}$ \\
\hline \multirow[t]{5}{*}{ PAD } & Training & $-.006(.004)$ & .124 & -.140 & .024 & $-.006(.004)$ & .155 & -.131 & .027 & $-.003(.005)$ & .576 & -.057 & .062 \\
\hline & State Mindfulness & $-.002(.002)$ & .334 & -.088 & & $-.002(.002)$ & .327 & -.089 & & $-.000(.003)$ & .958 & -.005 & \\
\hline & PME & & & & & $-.003(.005)$ & .516 & -.059 & & $.005(.009)$ & .594 & .079 & \\
\hline & Training x PME & & & & & & & & & $-.015(.011)$ & .190 & -.206 & \\
\hline & State Mindfulness x PME & & & & & & & & & $-.010(.006)$ & .116 & -.157 & \\
\hline \multirow[t]{5}{*}{ NAD } & Training & $-.002(.004)$ & .597 & -.049 & .002 & $-.002(.004)$ & 633 & -.045 & .003 & $-.002(.004)$ & .711 & -.038 & .032 \\
\hline & State Mindfulness & $-.000(.002)$ & .832 & -.019 & & $-.000(.002)$ & .827 & -.020 & & $.001(.002)$ & .563 & .059 & \\
\hline & PME & & & & & $-.001(.005)$ & .776 & -.026 & & $-.005(.008)$ & .569 & -.085 & \\
\hline & Training x PME & & & & & & & & & $.004(.010)$ & .723 & .056 & \\
\hline & State Mindfulness x PME & & & & & & & & & $-.011(.006)$ & .063 & -.189 & \\
\hline
\end{tabular}

Note. Step 1: Associations between PAD or NAD and mindfulness measures (Training and State mindfulness). Step 2: Controlled for PME. Step 3: Twoway interaction terms between PME and each mindfulness measure (Training and State mindfulness). PAD = Positive affect network density; NAD = Negative affect network density; State Mindfulness = grand-mean centered individual means of state Mindful Attention Awareness Scale reports; $\mathrm{PME}=$ Previous meditation experience $(0=$ no meditation or yoga experience; $1=$ previous meditation or yoga experience); Training $=$ condition allocation $(0=$ control condition; $1=$ mindfulness training $)$ 
Fig. 2 Estimated marginal means and $95 \%$ confidence intervals of overall affect (OA) network density for individuals with (solid line) and without (dash-dotted line) previous meditation experience who are either low $(-1 S D)$ or high $(+1 S D)$ in state mindfulness

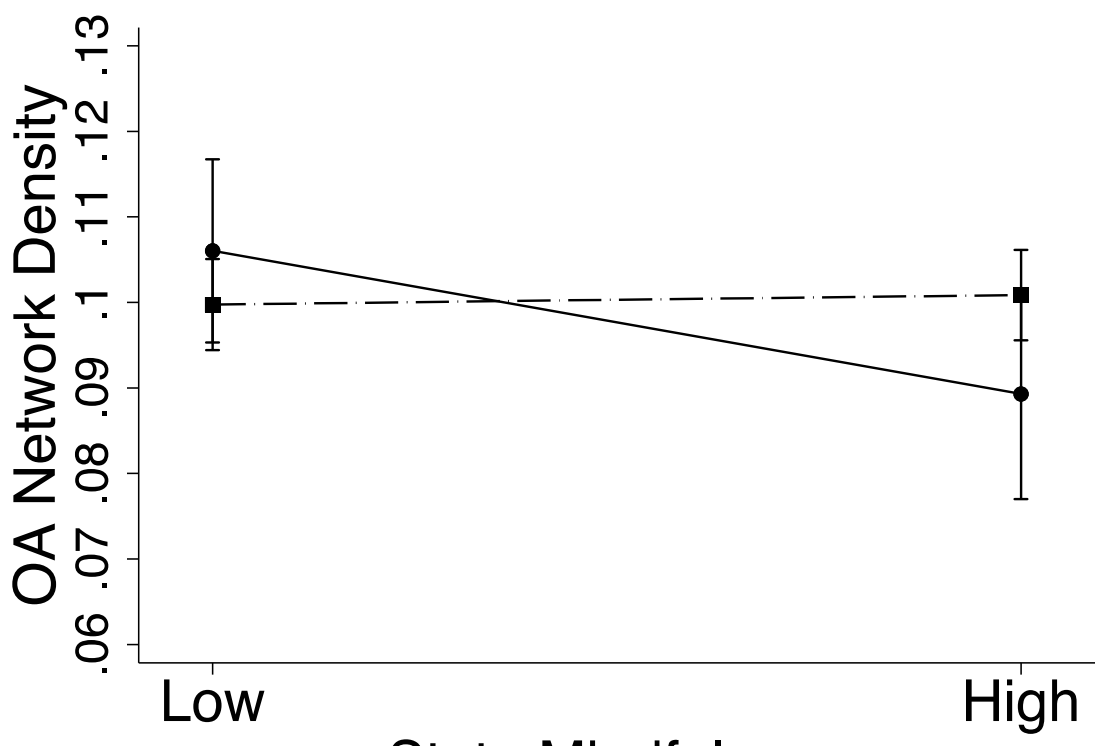

State Mindfulness

Finally, following the example of Pe et al. (2015), and to examine whether the found effects were consistent across the density of all negative and positive affective states, we also reported the associations between mindfulness measures and the density of each affective state in the Supplementary Material (all other previous affective states predicting a single affective state; Table S7). Consistent with our findings on $\mathrm{OAD}, \mathrm{PAD}$, and $\mathrm{NAD}$, we did not find a significant impact of the mindfulness training on any of the single affect network densities. The same pattern was found for the individual means of state mindfulness reports, with one exception: we found that the individual means of state mindfulness reports negatively predicted the density of the affective state relaxed, indicating that individuals who were on average more mindful in daily life experienced less impact of previous affective states on their present relaxed state than individuals who were less mindful ( $\beta=-.304, p=.001)$. We also found consistent small negative effects of the interaction between the individual means of state mindfulness reports and previous meditation experience on all single emotion network densities, although only the interaction effect on feeling anxious reached significance $(\beta=-.218, p=.029)$.

\section{Discussion}

The present study investigated whether a mindfulness training and state mindfulness helped people to disengage from prior affective experiences in daily life, which was assumed to represent a basic mechanism underlying the beneficial effects of mindfulness. The tendency to disengage from prior experiences was indexed by temporal network density (Bringmann et al. 2016b; Pe et al. 2015), with higher density values reflecting less ability to disengage from prior experiences.
We conducted a randomized controlled trial with a mindfulness training and a control condition, using an ambulatory assessment over a 40-day study period to assess momentary affective experiences and state mindfulness. On the basis of both previous laboratory studies (Britton et al. 2012; Cho et al. 2017) and recent ambulatory assessment studies that studied affective inertia (Keng and Tong 2016; Rowland et al. 2018) we assumed that everyday affective experiences of participants who train in mindfulness and who report high levels of state mindfulness would be more able to disengage from previous affective experiences in everyday life, as reflected by a less connected affect network, relative to participants who were in the control condition or who reported low levels of state mindfulness.

Contrary to our expectations, neither a brief mindfulness training nor state mindfulness showed significant associations with temporal affect network density. This is in line with a recent study by Snippe et al. (2017) who found that training in mindfulness had no significant impact on the affective system pre- to post-mindfulness intervention. Since network density may be particularly driven by either PA or NA network density rather than by a synergy of both affect valence networks (Pe et al. 2015), we also examined associations between mindfulness and PA and NA affect network density separately. As found for overall affect network density, these results did not indicate any associations between the mindfulness measures and either PA or NA network density. However, when additionally examining single-affect densities, we found that individuals who were more mindful experienced less impact of their previous affective states on their current state of feeling relaxed than individuals who reported to be less mindful. This is not surprising since particularly feeling relaxed may be interpreted as being closely related to the calm and accepting attitude that comes along with mindfulness (e.g., Desbordes 
et al. 2015; Lindsay and Creswell 2017). Thus, this finding may indicate that individuals who are mindful may not get wound up easily by previous affective experiences in that they are able to disengage from them, experiencing a relaxed and calm state in the present moment that is independent from prior experiences. Nevertheless, whereas laboratory-based findings (Britton et al. 2012; Cho et al. 2017) and research on affective inertia (e.g., Keng and Tong 2016; Rowland et al. 2018) have suggested that training mindfulness can help people to disengage from previous negative experiences, the present study may indicate that this is not necessarily the case when considering the complex affective system, which includes multiple different positive and negative affective states.

While inertia involves the self-predictability of a single affective state or an aggregated measure, density instead captures how several affective states are both self-predicted and predicted by all other affective states (Pe et al. 2015). Thus, the fact, that our results differ from those studies examining affective inertia, suggests that density is not solely driven by inertia, and that it represents more than how self-predictive affective states are. Furthermore, Pe et al. (2015) mentioned an additional important difference between inertia and network density: Since network density is indicated by the averaged absolute coefficient values of all temporal associations, both the direction and the negative or positive nature of the association between affective states is less important than they are for inertia, meaning that it does not matter how positive or negative associations between affective states are when examining network density. For example, two individuals may qualitatively differ in how their prior state of anger is associated with their following state of feeling relaxed: while one person may feel more relaxed, the other may feel less relaxed. Thus, the direction of the association may differ, but in both individuals the intensity of feeling relaxed may be similarly predicted by their prior anger. Thus, in both individuals we would find that their present state of relaxation could be predicted by their previous state of anger (this example is based on one used by Pe et al. 2015). This would indicate that they were not able to feel momentary relaxation regardless of what happened before. Instead of adapting present feelings to momentary situational circumstances, the intensity of feeling relaxed was determined by prior experiences in both individuals. In contrast, we assumed that a mindful individual would have been able to accept previous feelings so that present experiences may have been independent and less predictable by what had happened in the past. Therefore, the main findings that can be drawn from these explorations of network density is the degree of predictability and the fact that individuals differ in how predictable their affective states are (Pe et al. 2015).

Although we did not find any associations between mindfulness and affect network density, we further investigated whether the association between mindfulness and affective network density may be driven by previous meditation experiences. As Baer et al. (2008) found when validating the Five Facet Mindfulness Questionnaire (FFMQ; Baer et al. 2006), some mindfulness facets may only be associated with well-being in people with meditation experience, and not with individuals who merely self-report that they are more mindful than others. This speaks to a somewhat different understanding of mindfulness in experienced meditators as opposed to meditation novices. For this reason, we tested whether associations between mindfulness and affect network density may be moderated by meditation experience, finding that individuals who reported practicing yoga or meditation showed lower OA network density when they also reported high-state mindfulness, which also tended to apply to all single affect network densities. Thus, whereas individuals who indicated that they were mindful did not show any increased ability to disengage from previous affective experiences, yoga and meditation practitioners who reported being able to focus their attention on what was occurring in the present moment were more likely to disengage from previous affective experiences, relative to experienced mindfulness meditators who rated themselves to be low in mindfulness.

Several explanations may be helpful in understanding why the present-moment facet of mindfulness was found to be associated with affect network density, but only in participants experienced in practicing mindfulness. First, in some studies, neither the MAAS was found to differentiate between nonmeditators and novice meditators (MacKillop and Anderson 2007) nor the "acting with awareness" facet of the FFMQ, which is closely related to the MAAS, was positively associated with meditation experience (Baer et al. 2008). However, in contrast, Brown and Ryan (2003) found differences between Zen Buddhist practitioners and the general public using the MAAS.

Second, a study by Van Dam et al. (2009) found meditators to answer mindfulness items differently than non-meditators: While mindfulness meditators occurred to affirm mindfulness-absent and mindfulness-present items with about equal frequency, non-meditators more apparently tended to reject mindfulness-absent mindfulness items compared to affirming mindfulness-present items. Furthermore, even though non-meditators and meditators might happen to report similar levels on the present moment awareness facet of mindfulness (e.g., MacKillop and Anderson 2007) as measured with the MAAS (Brown and Ryan 2003), particularly individuals with meditation experience may have developed more skills, such as reacting calm, and nonjudgmentally accepting present affective experiences in daily life, in addition to only monitoring the present experiences (Lindsay and Creswell 2017). Thus, both novices and experienced meditators may be aware of their present experiences, but experienced meditators may find it easier to actually disengage from those experiences while novices may focus more on experiencing affective states, still judging them as pleasant 
or unpleasant, which in turn, may predict following affective experiences. In line with this, Desbordes et al. (2015) described that meditation beginners may first learn to monitor their experiences before applying acceptance in affectively loaded situations. Since we did not examine other mindfulness skills in daily life, such as nonjudgmentally accepting experiences and letting them pass by (e.g., Blanke and Brose 2017), this may be a plausible explanation for our main findings, which needs to be further investigated in future research by assessing discrete mindfulness skills in daily life.

Third, as Grossman (2011) mentioned, several qualities of the present-moment attention facet of mindfulness are not well represented in the currently available self-report measures, which tended to represent this subtle state more as an experience of inattentiveness (e.g., Brown and Ryan 2003). However, experienced meditators may not only see this state as inattentiveness, but rather as a deliberate choice to pay attention to the present moment, to investigate moment-tomoment experiences actively and with curiosity, and to be continuously aware of present-moment experiences (Grossman 2011). These aspects may be particularly crucial in disengaging from previous affective experiences.

Fourth, we can rule out the possibility that our findings are solely based on demand characteristics (e.g., Weber and Cook 1972). Since we assessed affect several times per day and we analyzed temporal associations between several items, it is unlikely that participants would have known what the researchers expected to find or how they expected them to behave, and would have therefore distorted their responses accordingly.

All of these considerations indicate that our findings may be based on the fact that experienced meditators and novices have different understandings of what present moment awareness actually is, and they may have different kinds of mindfulness skills that they can apply in daily life. Furthermore, mindfulness self-report questionnaires, such as the state version of the MAAS, may not be well suited to capturing these different qualities of mind.

\section{Limitations and Future Research}

Even though we did not find any evidence that either training mindfulness or state mindfulness were associated with affective network density, we found that mindfulness - and, in particular the individual means of present moment awareness reports that were assessed in daily life - was associated with lower network density in people who reported meditating or practicing yoga. However, several issues may limit the application of these findings.

First, we suggest caution when interpreting the results from our analyses hinting at a less interconnected affect network only in people who report higher mindfulness and who are experienced in meditation or yoga. Few participants indicated already practicing meditation or yoga before study participation, which limits our power to find small effects (e.g., NA and PA density). Furthermore, we did not assess how long individuals had practiced meditation or yoga. Thus, since this variable was only thought of as a control variable and was not the focus of our investigation, we cannot tell whether those individuals who answered "yes" continuously practiced mindfulness, or how long or to what degree they had been practicing. It would be interesting to further examine the association between actual mindfulness practice time and affect network density in experienced meditators to draw stronger conclusions on how the long-term practice of mindfulness may improve disengagement from previous experiences in daily life.

Second, we used a shortened version of the state version of the MAAS (Brown and Ryan 2003). This state mindfulness measure solely focused on assessing the level of inattentiveness on present activities. As other important aspects such as acceptance and nonjudgment (Lindsay and Creswell 2017) were not assessed, we cannot rule out the possibility that additional mindfulness aspects may be associated with network density. In recent studies, novel state mindfulness scales were constructed (e.g., Blanke and Brose 2017) and used (Friese and Hofmann 2016) that can assess mindfulness beyond a one-dimensional construct. Future research may choose multi-dimensional measures of state mindfulness to examine associations with affect network density.

Third, our mindfulness training sought to improve present moment awareness through a very simple breathing meditation of counting one's own breaths, and did not extend into additional, more advanced mindfulness skills (Bishop et al. 2004). Furthermore, our training sessions were short, and individuals had no opportunity to attend group sessions led by an experienced mindfulness meditators, which is standard practice for most mindfulness-based programs (e.g., MBSR; Kabat-Zinn 1990). However, we do not assume that this more extensive mindfulness training would have necessarily yielded different results, as the longer training implemented by Snippe et al. (2017) did not lead to any changes in affect network density from pre- to post-mindfulness intervention, relative to individuals in a control condition. Thus, it is possible that neither basic nor extensive mindfulness programs impact short-term affective network density. Rather, it seems that only continuous mindfulness practice over a long period may result in less interconnected affective networks. If this is the case, it may be that it is only through the frequent practice of mindfulness skills in daily life that meditators may increase disengagement from previous affective experiences.

Our fourth limitation is due to our network analyses: Although we had to assume stationarity of our time-series, we conducted a mindfulness training, which we expected to result in group differences in affect network density. Thus, although we tested for stationarity in our data, we cannot rule out that some individuals experienced changes in, for 
example, autocorrelations, since the Fisher-type test only tests against the hypothesis that all individual panels contain a unitroot (i.e., are non-stationary). We suspect, however, that our findings may serve as basis for further research on how mindfulness impacts affective networks. For example, Bringmann et al. (2016b) discussed other models, such as a multilevel threshold model (De Haan-Rietdijk et al. 2016) or a timevarying model (Bringmann et al. 2016a) that may be helpful in obtaining a deeper understanding of how mindfulness can help users to disengage from previous experiences.

Based on our presented findings, we assume that disengagement from previous affective experiences in daily life may take more practice than a short simple mindfulness training can provide. Additionally, the tendency to self-report being high in mindfulness does not necessarily indicate a stronger ability to disengage from experiences. Rather, only those who are already familiar with practicing mindfulness and who report being able to focus attention on the present moment seem to experience easier disengagement from previous affective experiences in their everyday lives. This speaks to a different understanding of mindfulness in long-term meditators who may already have applied a nonjudgmental and accepting attitude in their daily lives, in contrast to individuals who reported being high in mindfulness but who may have not applied this skill. Thus, we assume that multiple mindfulness practice sessions and a deep understanding of what mindfulness means may be needed to apply mindfulness effectively in daily life, ideally improving one's ability to disengage from previous experiences and to allow present-moment experiences to be more independent from what has happened in the past.

Acknowledgments This research was supported by a scholarship from the Stipendienstiftung Rheinland-Pfalz. We thank Prof. Dr. Thomas Kubiak who provided expertise that greatly assisted this research. We are also thankful to our research assistants Maike Vogel, Julius Welzel, Markus Müssig, and Kimberly Holtz who assisted us with study organization and data collection.

Author Contributions ZR: executed and organized the study, analyzed the data, and wrote the first draft of the manuscript. MW: designed and organized the study, assisted with the data analyses, and collaborated with the writing of the manuscript.

Funding Information Open Access funding provided by Projekt DEAL.

\section{Compliance with Ethical Standards}

Conflict of Interest The authors declare that they have no conflict of interest.

Ethical Approval This trial was approved by the local ethics committee at the Institute of Psychology at the Johannes Gutenberg-University of Mainz, Germany (2015-JGU-psychEK-011). All procedures performed in studies involving human participants were in accordance with the ethical standards of the institutional research committee Johannes
Gutenberg University Mainz and with the 1964 Helsinki declaration and its later amendments or comparable ethical standards.

Informed Consent Informed consent was obtained from all individual participants included in the study.

Data Availability Statement All data and syntax are available at the Open Science Framework (https://osf.io/jmz2n/).

Open Access This article is licensed under a Creative Commons Attribution 4.0 International License, which permits use, sharing, adaptation, distribution and reproduction in any medium or format, as long as you give appropriate credit to the original author(s) and the source, provide a link to the Creative Commons licence, and indicate if changes were made. The images or other third party material in this article are included in the article's Creative Commons licence, unless indicated otherwise in a credit line to the material. If material is not included in the article's Creative Commons licence and your intended use is not permitted by statutory regulation or exceeds the permitted use, you will need to obtain permission directly from the copyright holder. To view a copy of this licence, visit http://creativecommons.org/licenses/by/4.0/.

\section{References}

Baer, R. A., Smith, G. T., Hopkins, J., Krietemeyer, J., \& Toney, L. (2006). Using self-report assessment methods to explore facets of mindfulness. Assessment, 13(1), 27-45. https://doi.org/10.1177/ 1073191105283504

Baer, R. A., Smith, G. T., Lykins, E., Button, D., Krietemeyer, J., Sauer, S., et al. (2008). Construct validity of the five facet mindfulness questionnaire in meditating and nonmeditating samples. Assessment, 15(3), 329-342. https://doi.org/10.1177/ 1073191107313003 .

Bishop, S. R., Lau, M., Shapiro, S., Carlson, L., Anderson, N. D., Carmody, J., et al. (2004). Mindfulness: a proposed operational definition. Clinical Psychology: Science and Practice, 11(3), 230-241. https://doi.org/10.1093/clipsy/bph077.

Blanke, E. S., \& Brose, A. (2017). Mindfulness in daily life: a multidimensional approach. Mindfulness, 8(3), 737-750. https://doi.org/10. 1007/s12671-016-0651-4.

Borsboom, D., \& Cramer, A. O. J. (2013). Network analysis: an integrative approach to the structure of psychopathology. Annual Review of Clinical Psychology, 9, 92-121. https://doi.org/10.1146/annurevclinpsy-050212-185608.

Bringmann, L. F., Vissers, N., Wichers, M., Geschwind, N., Kuppens, P., Peeters, F., et al. (2013). A network approach to psychopathology: new insights into clinical longitudinal data. PLoS One, 8(4), e60188. https://doi.org/10.1371/journal.pone.0060188.

Bringmann, L. F., Hamaker, E. L., Vigo, D. E., Aubert, A., Borsboom, D., \& Tuerlinckx, F. (2016a). Changing dynamics: time-varying autoregressive models using generalized additive modeling. Psychological Methods, 22(3), 409-425. https://doi.org/10.1037/ met0000085.

Bringmann, L. F., Pe, M. L., Vissers, N., Ceulemans, E., Borsboom, D., Vanpaemel, W., et al. (2016b). Assessing temporal emotion dynamics using networks. Assessment, 23(4), 425-435. https://doi.org/10. 1177/1073191116645909.

Britton, W. B., Shahar, B., Szepsenwol, O., \& Jacobs, W. J. (2012). Mindfulness-based cognitive therapy improves emotional reactivity to social stress: results from a randomized controlled trial. Behavior Therapy, 43(2), 365-380. https://doi.org/10.1016/j.beth.2011.08. 006. 
Broderick, P. C. (2005). Mindfulness and coping with dysphoric mood: contrasts with rumination and distraction. Cognitive Therapy and Research, 29(5), 501-510. https://doi.org/10.1007/s10608-0053888-0.

Brown, K. W., \& Ryan, R. M. (2003). The benefits of being present: mindfulness and its role in psychological well-being. Journal of Personality and Social Psychology, 84(4), 822-848. https://doi. org/10.1037/0022-3514.84.4.822.

Carmody, J., \& Baer, R. A. (2008). Relationships between mindfulness practice and levels of mindfulness, medical and psychological symptoms and well-being in a mindfulness-based stress reduction program. Journal of Behavioral Medicine, 31(1), 23-33. https://doi. org/10.1007/s10865-007-9130-7.

Chen, J., \& Chen, Z. (2008). Extended Bayesian information criteria for model selection with large model spaces. Biometrika, 95(3), 759 771. https://doi.org/10.1093/biomet/asn034.

Cho, S., Lee, H., Oh, K. J., \& Soto, J. A. (2017). Mindful attention predicts greater recovery from negative emotions, but not reduced reactivity. Cognition and Emotion, 31(6), 1252-1259. https://doi. org/10.1080/02699931.2016.1199422.

Choi, I. (2001). Unit root tests for panel data. Journal of International Money and Finance, 20(2), 249-272. https://doi.org/10.1016/ S0261-5606(00)00048-6.

De Haan-Rietdijk, S., Gottman, J. M., Bergeman, C. S., \& Hamaker, E. L. (2016). Get over it! A multilevel threshold autoregressive model for state-dependent affect regulation. Psychometrika, 81(1), 217-241. https://doi.org/10.1007/s11336-014-9417-x.

Desbordes, G., Gard, T., Hoge, E. A., Hölzel, B. K., Kerr, C., Lazar, S. W., et al. (2015). Moving beyond mindfulness: defining equanimity as an outcome measure in meditation and contemplative research. Mindfulness, 6(2), 356-372. https://doi.org/10.1007/s12671-0130269-8.

Epskamp, S. (2018). graphicalVAR: Graphical VAR for experience sampling data. $R$ package version 0.2.2. https://CRAN.R-project.org/ package $=$ graphicalVAR .

Epskamp, S., Cramer, A. O. J., Waldorp, L. J., Schmittmann, V. D., \& Borsboom, D. (2012). qgraph: Network visualizations of relationships in psychometric data. Journal of Statistical Software, 48(4), 118 URL http://www.jstatsoft.org/v48/i04/.

Epskamp, S., Waldorp, L. J., Mõttus, R., \& Borsboom, D. (2018). The Gaussian graphical model in cross-sectional and time-series data. Multivariate Behavioral Research, 53(4), 453-480. https://doi.org/ 10.1080/00273171.2018.1454823.

Friese, M., \& Hofmann, W. (2016). State mindfulness, self-regulation, and emotional experience in everyday life. Motivation Science, 2(1), 1-14. https://doi.org/10.1037/mot0000027.

Frijda, N. H. (2007). The laws of emotion. Mahwah: Lawrence Erlbaum Associates Publishers.

Grossman, P. (2011). Defining mindfulness by how poorly I think I pay attention during everyday awareness and other intractable problems for psychology's (re)invention of mindfulness: Comment on Brown et al. (2011). Psychological Assessment, 23(4), 1034-1040. https:// doi.org/10.1037/a0022713.

Gruber, R., \& Cassoff, J. (2014). The interplay between sleep and emotion regulation: Conceptual framework empirical evidence and future directions. Current Psychiatry Reports, 16, 500. https://doi.org/ 10.1007/s11920-014-0500-x.

Houben, M., Van Den Noortgate, W., \& Kuppens, P. (2015). The relation between short-term emotion dynamics and psychological well-being: a meta-analysis. Psychological Bulletin, 141(4), 901-930. https://doi.org/10.1037/a0038822.

Kabat-Zinn, J. (1990). Full catastrophe living: using the wisdom of your body and mind to face stress, pain and illness. New York, NY: Delacorte Press.

Keng, S.-L., \& Tong, E. M. W. (2016). Riding the tide of emotions with mindfulness: mindfulness, affect dynamics, and the mediating role of coping. Emotion, 16(5), 706-718. https://doi.org/10.1037/ emo0000165.

Khoury, B., Lecomte, T., Fortin, G., Masse, M., Therien, P., Bouchard, V., et al. (2013). Mindfulness-based therapy: a comprehensive metaanalysis. Clinical Psychology Review, 33(6), 763-771. https://doi. org/10.1016/j.cpr.2013.05.005.

Khoury, B., Sharma, M., Rush, S. E., \& Fournier, C. (2015). Mindfulness-based stress reduction for healthy individuals: a metaanalysis. Journal of Psychosomatic Research, 78(6), 519-528. https://doi.org/10.1016/j.jpsychores.2015.03.009.

Koval, P., Kuppens, P., Allen, N. B., \& Sheeber, L. (2012). Getting stuck in depression: the roles of rumination and emotional inertia. Cognition \& Emotion, 26(8), 1412-1427. https://doi.org/10.1080/ 02699931.2012.667392.

Koval, P., Brose, A., Pe, M. L., Houben, M., Erbas, Y., Champagne, D., \& Kuppens, P. (2015). Emotional inertia and external events: the roles of exposure, reactivity, and recovery. Emotion, 15(5), 625-636. https://doi.org/10.1037/emo0000059.

Kuppens, P., Allen, N. B., \& Sheeber, L. B. (2010). Emotional inertia and psychological maladjustment. Psychological Science, 21(7), 984 991. https://doi.org/10.1177/0956797610372634.

Kuyken, W., Byford, S., Taylor, R. S., Watkins, E., Holden, E., White, K., et al. (2008). Mindfulness-based cognitive therapy to prevent relapse in recurrent depression. Journal of Consulting and Clinical Psychology, 76(6), 966-978. https://doi.org/10.1037/a0013786.

Levinson, D. B., Stoll, E. L., Kindy, S. D., Merry, H. L., \& Davidson, R. J. (2014). A mind you can count on: validating breath counting as a behavioral measure of mindfulness. Frontiers in Psychology, 5, 1202. https://doi.org/10.3389/fpsyg.2014.01202.

Lindsay, E. K., \& Creswell, J. D. (2017). Mechanisms of mindfulness training: monitor and acceptance theory (MAT). Clinical Psychology Review, 51, 48-59. https://doi.org/10.1016/j.cpr.2016. 10.011 .

MacKillop, J., \& Anderson, E. J. (2007). Further psychometric validation of the mindful attention awareness scale (MAAS). Journal of Psychopathology and Behavioral Assessment, 29(4), 289-293. https://doi.org/10.1007/s10862-007-9045-1.

Newman, M. E. J. (2010). Networks: an introduction. New York, NY: Oxford University Press.

Pe, M. L., \& Kuppens, P. (2012). The dynamic interplay between emotions in daily life: augmentation, blunting, and the role of appraisal overlap. Emotion, 12(6), 1320-1328. https://doi.org/10.1037/ a0028262.

Pe, M. L., Kircanski, K., Thompson, R. J., Bringmann, L. F., Tuerlinckx, F., Mestdagh, M., et al. (2015). Emotion-network density in major depressive disorder. Clinical Psychological Science, 3(2), 292-300. https://doi.org/10.1177/2167702614540645.

R Core Team. (2019). R: a language and environment for statistical computing. R Foundation for statistical computing, Vienna, Austria. URL http://www.R-project.org/.

Roelofs, J., Huibers, M., Peeters, F., Arntz, A., \& Van Os, J. (2008). Rumination and worrying as possible mediators in the relation between neuroticism and symptoms of depression and anxiety in clinically depressed individuals. Behaviour Research and Therapy, 46(12), 1283-1289. https://doi.org/10.1016/j.brat.2008.10.002.

Rowland, Z., Wenzel, M., \& Kubiak, T. (2016). The effects of computerbased mindfulness training on self-control and mindfulness within ambulatorily assessed network systems across health-related domains in a healthy student population (SMASH): study protocol for a randomized controlled trial. Trials, 17, 570. https://doi.org/ 10.1186/s13063-016-1707-4.

Rowland, Z., Wenzel, M., \& Kubiak, T. (2018). A mind full of happiness: How mindfulness shapes affect dynamics in daily life. Emotion. Advance online publication. https://doi.org/10.1037/emo0000562.

Rowland, Z., Wenzel, M., \& Kubiak, T. (2019). Effects of an ultra-brief computer-based mindfulness training on mindfulness and self- 
control: a randomised controlled trial using a 40-day ecological momentary assessment. Mindfulness, 10(11), 2312-2326. https://doi. org/10.1007/s12671-019-01204-9.

Russell, J. A. (2003). Core affect and the psychological construction of emotion. Psychological Review, 110(1), 145-172. https://doi.org/10. 1037/0033-295X.110.1.145.

Segal, Z. V., Williams, J. M. G., \& Teasdale, J. D. (2002). Mindfulnessbased cognitive therapy for depression: a new approach to preventing relapse. New York, NY: Guilford Press.

Semple, R. J., Lee, J., Rosa, D., \& Miller, L. F. (2010). A randomized trial of mindfulness-based cognitive therapy for children: promoting mindful attention to enhance social-emotional resiliency in children. Journal of Child and Family Studies, 19(2), 218-229. https://doi. org/10.1007/s10826-009-9301-y.

Shiffman, S., Stone, A. A., \& Hufford, M. R. (2008). Ecological momentary assessment. Annual Review of Clinical Psychology, 4, 1-32. https://doi.org/10.1146/annurev.clinpsy.3.022806.091415.

Snippe, E., Viechtbauer, W., Geschwind, N., Klippel, A., de Jonge, P., \& Wichers, M. (2017). The impact of treatments for depression on the dynamic network structure of mental states: two randomized controlled trials. Scientific Reports, 7, 46523. https://doi.org/10.1038/ srep46523.

StataCorp. (2017). Stata statistical software: release 15. College Station, TX: StataCorp LLC.

Suls, J., Green, P., \& Hillis, S. (1998). Emotional reactivity to everyday problems, affective inertia, and neuroticism. Personality and Social Psychology Bulletin, 24(2), 127-136. https://doi.org/10.1177/ 0146167298242002.

Tibshirani, R. (1996). Regression shrinkage and selection via the lasso. Journal of the Royal Statistical Society Series B (Methodological), 58(1), 267-288.

Trull, T. J., \& Ebner-Priemer, U. (2013). Ambulatory assessment. Annual Review of Clinical Psychology, 9, 151-176. https://doi.org/10.1146/ annurev-clinpsy-050212-185510.

Van Dam, N. T., Earleywine, M., \& Danoff-Burg, S. (2009). Differential item function across meditators and non-meditators on the five facet mindfulness questionnaire. Personality and Individual Differences, 47(5), 516-521. https://doi.org/10.1016/j.paid.2009.05.005.

Weber, S. J., \& Cook, T. D. (1972). Subject effects in laboratory research: an examination of subject roles, demand characteristics, and valid inference. Psychological Bulletin, 77(4), 273-295. https://doi.org/ 10.1037/h0032351.

Wenzel, M., \& Kubiak, T. (2018). Ambulatory monitoring and ambulatory assessment in personality research. In V. Zeigler-Hill \& T. K. Shackelford (Eds.), The SAGE handbook of personality and individual differences: The science of personality and individual differences (pp. 305-316). Sage Reference. https://doi.org/10. 4135/9781526451163.n14.

Wichers, M. (2014). The dynamic nature of depression: a new microlevel perspective of mental disorder that meets current challenges. Psychological Medicine, 44, 1349-1360. https://doi.org/10.1017/ S0033291713001979.

Wigman, J. T. W., Van Os, J., Borsboom, D., Wardenaar, K. J., Epskamp, S., Klippel, A., et al. (2015). Exploring the underlying structure of mental disorders: cross-diagnostic differences and similarities from a network perspective using both a top-down and a bottom-up approach. Psychological Medicine, 45(11), 2375-2387. https://doi. org/10.1017/S0033291715000331.

Wild, B., Eichler, M., Friedrich, H., Hartmann, M., Zipfel, S., \& Herzog, W. (2010). A graphical vector autoregressive modelling approach to the analysis of electronic diary data. BMC Medical Research Methodology, 10(28), 1-13. https://doi.org/10.1186/1471-2288-1028.

World Medical Association. (2013). Declaration of Helsinki: ethical principles for medical research involving human subjects. JAMA, 310(20), 2191-2194. https://doi.org/10.1001/jama.2013.281053.

Publisher's Note Springer Nature remains neutral with regard to jurisdictional claims in published maps and institutional affiliations. 